\title{
"Successful Conservative Management of a Large Traumatic Mesenteric Hematoma: A Case Report"
}

\author{
Sunil Basukala ${ }^{1}$, Ayush Tamang ${ }^{2,3}$, Shriya Sharma ${ }^{3}$, Ujwal Bhusal $^{3}$, and Bishnu Pathak ${ }^{3}$ \\ ${ }^{1}$ Shree Birendra Hospital \\ 2a.tmng@outlook.com \\ ${ }^{3}$ Nepalese Army Institute of Health Sciences
}

January 25, 2022

\begin{abstract}
INTRODUCTION AND IMPORTANCE: In blunt abdominal trauma, small bowel and mesentery injury (SBMI) is the third most common organ injury with an estimated incidence of 1-5\%. Traumatic mesenteric injuries are difficult to diagnose and their undiagnosed complications are severe with high mortality rates.

CASE PRESENTATION: A 21-year-old male presented to the emergency room with severe colicky pain in the right-hypochondrium two hours after dinner. He gave a history of nausea, vomiting and diarrhea after dinner and before the pain started. The morning after admission, a Contrast Enhanced Computed Tomography (CECT) scan was done which showed a large mesenteric hematoma. On subsequent questioning, our patient then recalled a blow to the abdomen while playing basketball two days ago. Since the patient was hemodynamically stable, non-operative management (NOM) was chosen with close monitoring. Regular follow-up ultrasonography (USG) scans showed progressive spontaneous resolution of the hematoma.

CLINICAL DISCUSSION: Nonspecific symptoms of mesenteric hematoma make it difficult to reach a diagnosis. It is usually identified by history or medical imaging. Mesenteric hematoma can be managed conservatively if there is no active bleeding. In stable patients, selective visceral angiography should be performed and bleeding vessels should be embolized where possible.

CONCLUSION: Our case highlights successful conservative management of a large traumatic mesenteric hematoma. This case emphasizes the importance of eliciting a detailed history of major or minor trauma for any patient with abdominal pain. Previous cases have also highlighted the importance of non-operative management and avoidance of emergency laparotomy in stable patients.
\end{abstract}

\section{INTRODUCTION}

In blunt abdominal trauma, small bowel and mesentery injury (SBMI) is the third most common organ injury with an estimated incidence of 1-5\% [1,2]. Isolated SBMI lesions are uncommon and delayed diagnosis is often frequent, with increased morbidity and mortality [3]. Mesenteric injury following trauma is relatively rare. However, it is clinically important because it can be associated with intraperitoneal, gastrointestinal, or retroperitoneal bleeding, intestinal ischemia, stenosis, and/or perforation [4]. We report a rare case of a large mesenteric hematoma following blunt abdominal trauma with successful conservative management.

We report this case in line with the updated consensus-based surgical case report (SCARE) guidelines [5].

\section{CASE PRESENTATION}

A 21-year-old medical student presented to the emergency room with severe colicky abdominal pain at right hypochondrium two hours after dinner. The patient gave history of an episode of diarrhea before the onset of pain abdomen. There was no history of melena or hematemesis. He also had nausea and two episodes of vomiting following the diarrhea. Then, the abdominal pain started shortly afterwards which was 
intermittently severe and non-migratory. He took no medication and had no allergies. There was no family history of bleeding diathesis.

On presentation, he was drowsy and afebrile with blood pressure of 110/65 mm Hg and pulse of 98 beats/min. On examination, the abdomen was soft but had a right-sided fullness and tenderness. Based on the presentation and similar history of food poisoning two months ago, acute gastroenteritis (AGE) was suspected. The patient received IV antibiotics, analgesics and fluid resuscitation. Initial blood report showed a hemoglobin $(\mathrm{Hb})$ of $11.2 \mathrm{~g} / \mathrm{dL}$, white blood cells of $14 \times 109 / \mathrm{L}$, with normal platelet count and amylase level. On Arterial Blood Gas (ABG) analysis the lactate was $2.0 \mathrm{mmol} / \mathrm{L}$, the $\mathrm{pH}$ was 7.30 . Fluid resuscitation continued with $2000 \mathrm{ml}$ of fluids in the first 3 hours.

The next morning, the patient still complained of colicky abdominal pain. His hemoglobin had dropped to $10.8 \mathrm{~g} / \mathrm{dL}$ in the morning. Contrast Enhanced Computed Tomography (CECT) revealed a large mesenteric hematoma in the right iliac fossa measuring approximately $8.2 \times 6.2 \times 8.7 \mathrm{~cm}$. The hematoma had a central hyperdense focus probably due to an injured branch of Ileocolic artery (Figure 1). On subsequent questioning, the patient recalled a blow to the right hypochondrium while playing basketball two days back. During the game, our patient had jumped and his abdomen landed on the opponent's shoulder which was being driven forward and up. He, however, mentioned that the pain from the impact was transient and continued playing afterwards. Hence, he did not recall the incident at the day of admission.

Patient was admitted in the Intensive Care Unit (ICU). Further selective visceral angiography of the coeliac trunk/superior mesenteric artery was done which showed no source of bleeding and therefore no embolization was required. The patient was treated with conservative management -intravenous fluids, analgesics, antiemetics and Tranexamic acid injection. The patient's hemoglobin level showed improvement to $12.0 \mathrm{~g} / \mathrm{dL}$ and $14.5 \mathrm{~g} / \mathrm{dL}$ during the fourth and fifth day after trauma, respectively. Ultrasonography (USG) showed a decreased size of the hematoma on the fifth day after trauma. Once the patient's condition improved, he was sent home with Omeprazole (dosage: $40 \mathrm{mg}$ once daily) and Ferrous sulphate tablets (dosage: $200 \mathrm{mg}$ thrice daily). The patient was counseled to restrict strenuous physical activities for at least two months.

He was called for follow-up two, four and twelve weeks after discharge in the surgical outpatient department. The mesenteric hematoma showed a progressively smaller size on follow-up USG. It resolved spontaneously and was undetectable via USG by the 12-week follow-up period. 


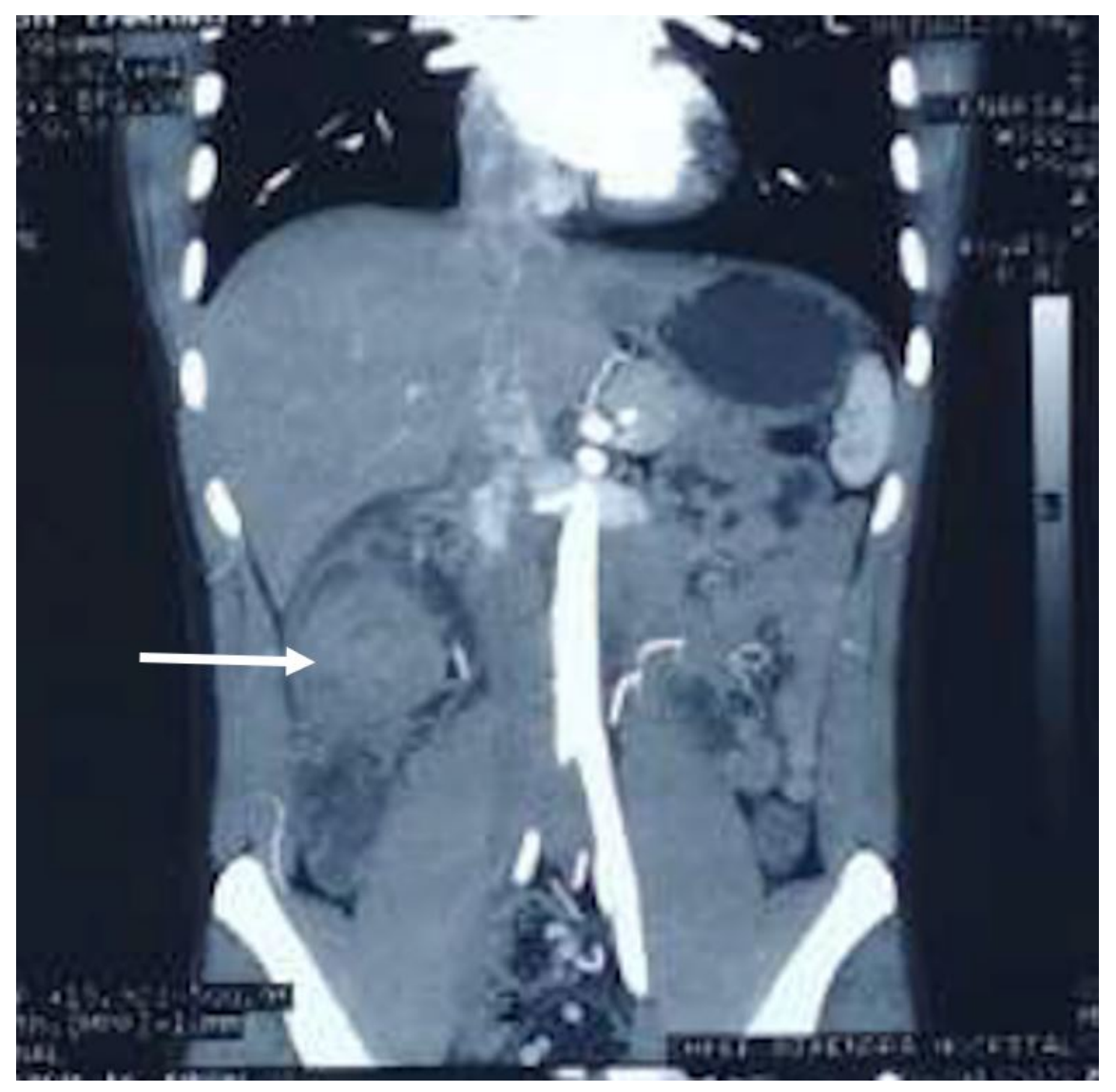

Figure 1: Contrast Enhanced Computed Tomography (CECT) of the abdomen showing mesenteric hematoma in right iliac fossa. 


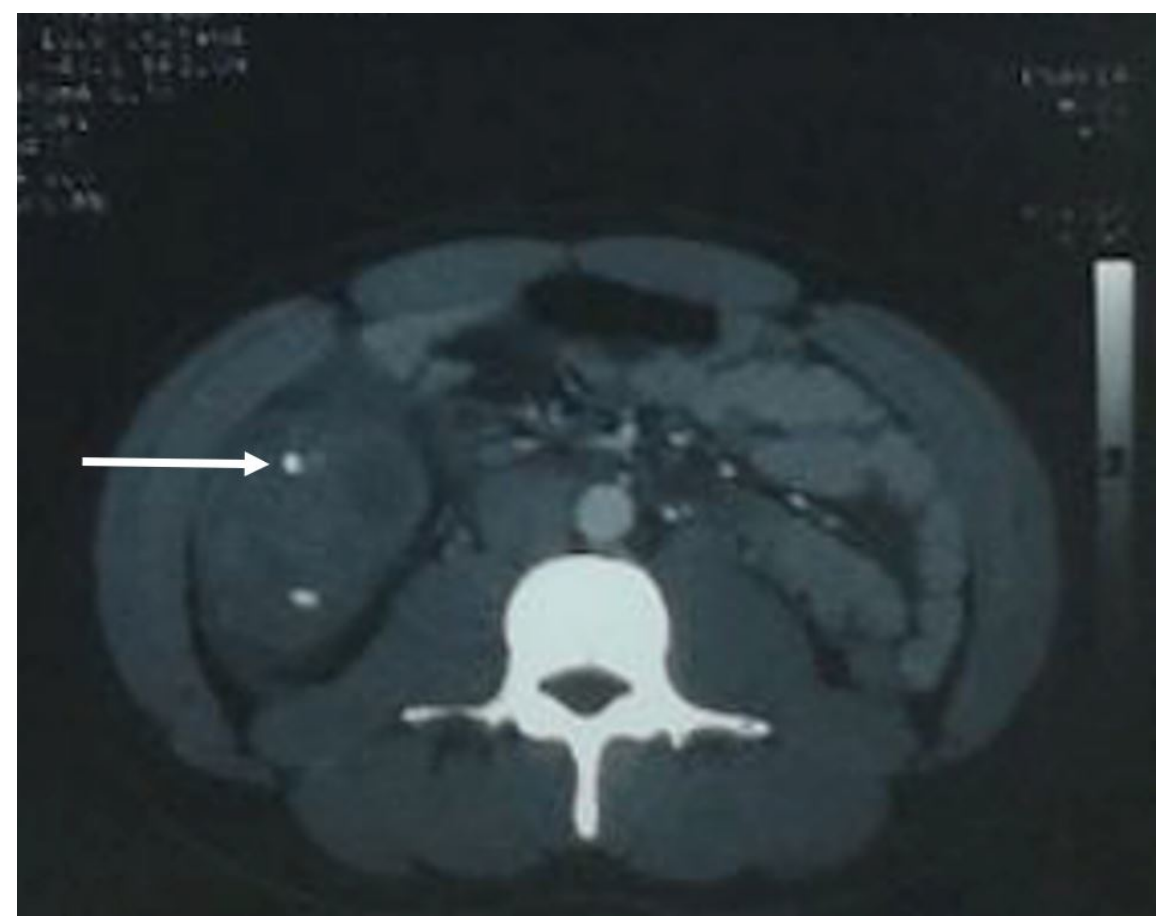

Figure 2: Contrast Enhanced Computed Tomography (CECT) of the abdomen confirmed mesenteric bleed with contrast extravasation.

The timeline of events and interventions is summarized in the following diagram (Figure 3).

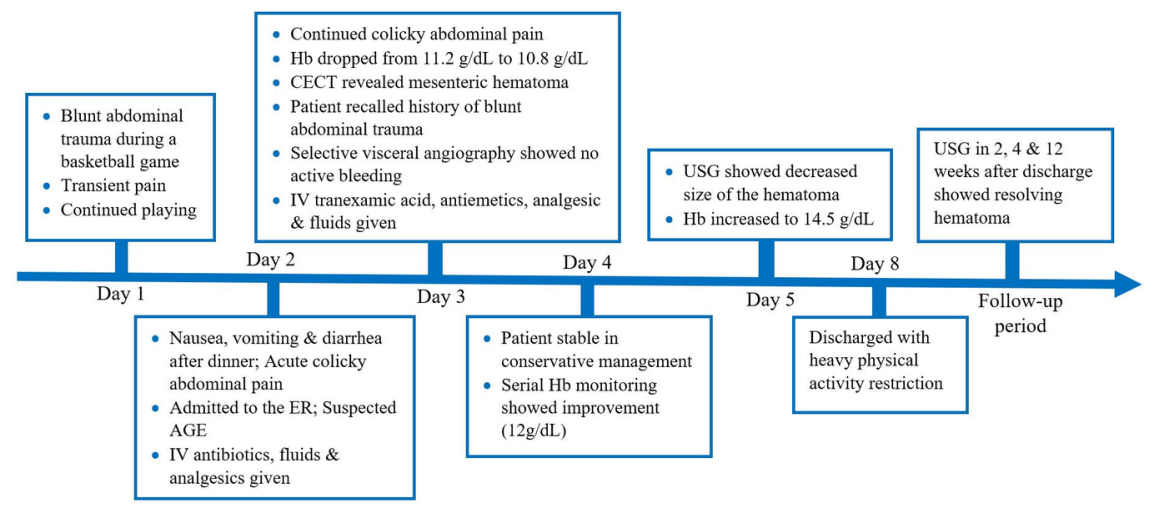

Figure 3: Timeline of events and interventions (ER: emergency room; AGE: acute gastroenteritis; IV: intravenous; CECT: contrast enhanced computed tomography; Hb: hemoglobin; USG: ultrasonography).

\section{DISCUSSION}

Mesenteric hematoma is a rare condition due to a localized bleeding from peripheral mesenteric vessels that is generally caused by abdominal trauma, postoperative complications, or aneurysm [6]. The most common clinical symptom is abdominal pain, the severity of which depends on the location and size of the hematoma. Nausea or constipation may occur if the tumor is large enough to compress the digestive tract $[7,8]$. Nonspecific symptoms make it difficult to reach a diagnosis and mesenteric hematoma is usually 
identified by history, abdominal CECT, ultrasound, or magnetic resonance imaging (MRI) [9]. CT scanning is the standard imaging modality which is required to rule out other more common causes of abdominal pain and shock; including abdominal aneurysm, malignancy and acute pancreatitis.

The morning after admission, the patient still had abdominal pain and a decrease in Hb level. Then, a CECT scan was done which showed a large mesenteric hematoma. On subsequent questioning, our patient then recalled the incident of blunt abdominal trauma. He mentioned that the pain was transient and did not remember the incident as significant. This shows the importance of eliciting a detailed history of major or minor trauma for any patients with abdominal pain, as highlighted in other cases too [10].

Management of patients with mesenteric hematoma depends on their clinical stability. Patients in shock not responding to fluid resuscitation need an emergency operation. Patients stable after resuscitation require urgent imaging. If the patient is stable and the CT is suggestive of a mesenteric hematoma, selective visceral angiography should be performed. Where possible, bleeding vessels should be embolized [11]. Recent studies have shown embolization to be successful in the treatment of ruptured aneurysm secondary to pancreatitis and in postoperative bleeding events $[12,13]$.

This case demonstrates a successful non-operative management of a large mesenteric hematoma in a clinically stable patient. Neither CT scan nor visceral angiography could identify any active bleeding or cause for hemorrhage. Previous cases have also highlighted the importance of non-operative management and avoidance of emergency laparotomy in stable patients [10]. Regular imaging and clinical follow-up are required to make sure the hematoma is reducing in size and the patient remains well.

\section{CONCLUSION}

We present a case of successful conservative management of a large traumatic mesenteric hematoma. This case highlights the importance of eliciting a detailed history of major or minor trauma for any patients with abdominal pain. If the patient is stable and the $\mathrm{CT}$ is suggestive of a mesenteric hematoma, selective visceral angiography should be performed and bleeding vessels embolized, where possible.

\section{CONFLICT OF INTEREST}

None declared.

\section{AUTHOR CONTRIBUTIONS}

Sunil Basukala $(\mathrm{SB})=$ Visualization, Supervision

Ayush Tamang (AT), Shriya Sharma (SS) = Writing - original draft

SB, AT, SS, Ujwal Bhusal (UB), Bishnu Pathak (BP) = Writing - review \& editing

All the authors read and approved the final manuscript.

ETHICAL APPROVAL: All procedures performed in studies involving human participants were done in accordance with the ethical standards of the institutional and/or national research committee and with the 1964 Helsinki Declaration and its later amendments or comparable ethical standards.

\section{CONSENT STATEMENT}

Written informed consent was obtained from the patient for publication of this case report and accompanying images. A copy of the written consent is available for review by the Editor-in-Chief of this journal on request.

\section{DATA AVAILABILITY STATEMENT}

Data sharing is not applicable to this article as no datasets were generated or analyzed during the current study.

\section{REFERENCES}


1. V.U.G. Vivek, M.D. Blair, S. Adnan, Small-bowel and mesenteric injuries in blunt trauma of the abdomen, Can. Assoc. Radiol. J. 64 (2013) 140-147. doi.org/10.1016/j.carj.2012.10.001

2. S.M. Fakhry, M. Brownstein, D.D. Watts, C.C. Baker, D. Oller, Relatively short diagnostic delays ( $<8$ hours) produce morbidity and mortality in blunt small bowel injury: an analysis of time to operative intervention in 198 patients from a multicenter experience, J. Trauma 48 (3) (2000) 408-414 doi.org/10.1097/00005373-200003000-00007

3. P. Addeo, D.P. Calabrese, Diagnostic and therapeutic value of laparoscopy for small bowel blunt injuries: a case report, Int. J. Surg. Case Rep. 2 (8) (2011) 316-318. doi.org/10.1016/j.ijscr.2011.10.005

4. Asayama Y, Matsumoto S, Isoda T, Kunitake N, Nakashima H. A case of traumatic mesenteric bleeding controlled by only transcatheter arterial embolization. Cardiovasc Intervent Radiol 2005; 28:256258. doi.org/10.1007/s00270-003-9108-y

5. 5. R.A. Agha, T. Franchi, C. Sohrabi, G. Mathew, A. Kerwan, A. Thoma, A.J. Beamish, A. Noureldin, A. Rao, B. Vasudevan, B. Challacombe, B. Perakath, B. Kirshtein, B. Ekser, C.S. Pramesh, D.M. Laskin, D. Machado-Aranda, D. Miguel, D. Pagano, F.H. Millham, G. Roy, H. Kadioglu, I.J. Nixon, I. Mukherjee, J.A. McCaul, J. Chi-Yong Ngu, J. Albrecht, J.G. Rivas, K. Raveendran, L. Derbyshire, M.H. Ather, M.A. Thorat, M. Valmasoni, M. Bashashati, M. Chalkoo, N.Z. Teo, N. Raison, O.J. Muensterer, P.J. Bradley, P. Goel, P.S. Pai, R.Y. Afifi, R.D. Rosin, R. Coppola, R. Klappenbach, R. Wynn, R.L. De Wilde, S. Surani, S. Giordano, S. Massarut, S.G. Raja, S. Basu, S.A. Enam, T.G. Manning, T. Cross, V.K.L. Karanth, V. Kasivisvanathan, Z. Mei, The SCARE 2020 Guideline: Updating Consensus Surgical CAse REport (SCARE) Guidelines, Int. J. Surg. 84 (2020) 226-230. https://doi.org/https://doi.org/10.1016/j.ijsu.2020.10.034.

6. Meissnitzer MW, Stättner S, Meissnitzer T. Small mesenteric hematoma following blunt abdominal trauma as early sign in computed tomography of occult small bowel perforation-report of 2 cases. Emerg Radiol. 2014;21: 647-50. doi.org/10.1007/s10140-014-1235-z

7. Parker SG, Thompson JN. Spontaneous mesenteric haematoma; diagnosis and management. BMJ Case Rep. 2012;2012. doi.org/10.1136/bcr-2012-006624

8. Hirano K, Bando T, Osawa S, Shimizu T, Okumura T, Fujii T. Spontaneous mesenteric hematoma of the sigmoid colon associated with rivaroxaban: a case report. Int J Surg Case Rep. 2018;44:33 doi.org/10.1016/j.ijscr.2018.02.008

9. Virmani V, George U, MacDonald B, Sheikh A. Small bowel and mesenteric injuries in blunt trauma of the abdomen. Can Assoc Radiol J 2013; 64:140-147

10. S. Basukala, A. Tamang, U. Bhusal, S. Sharma, B. Karki, Delayed splenic rupture following trivial trauma: A case report and review of literature, Int. J. Surg. Case Rep. 88 (2021) 106481. https://doi.org/https: //doi.org/10.1016/j.ijscr.2021.106481.

11. Pathi R, Voyvodic F, Thompson WR. Spontaneous extraperitoneal haemorrhage: computed tomography diagnosis and treatment by selective arterial embolisation. J Aust Radiol 2004;48:1238. doi.org/10.1111/j.1440-1673.2004.01271.x

12. Gomez D, Rahman SH, Guillou PJ. Spontaneous mesenteric haematoma: a disagnostic challenge. Ann R Coll Surg Engl 2006;88:269. doi.org/10.1308/147870806x95230

13. N Brofman M Atri JM Hanson L Grinblat T Chughtai F Brenneman (2006) Evaluation of bowel and mesenteric blunt trauma with multidetector CT Radiographics 26 1119-31 doi.org/10.1148/rg.264055144

\section{Hosted file}

Figures.docx available at https://authorea.com/users/437606/articles/539122--successfulconservative-management-of-a-large-traumatic-mesenteric-hematoma-a-case-report 